\title{
Tensions and Contradictions in Interns' Communication about Unexpected Pregnancy Loss
}

\author{
Jennifer J. Bute \& Maria Brann
}

\begin{abstract}
Early miscarriage is an unexpected pregnancy complication that affects up to $25 \%$ of pregnant women. Physicians are often tasked with delivering the bad news of a pregnancy loss to asymptomatic women while also helping them make an informed decision about managing the miscarriage. Assessing the communicative responses, particularly the discursive tensions embedded within providers' speech, offers insight into the (in)effective communication used in the delivery of bad news and the management of a potentially traumatic medical event. We observed and analyzed transcripts from 40 standardized patient encounters using Baxter's relational dialectics theory 2.0. Results indicated that interns invoked two primary distal alreadyspoken discourses: discourses of medicalization of miscarriage and discourses of rationality and informed consent. We contend that tensions and contradictions could affect how women respond to the news of an impending miscarriage and offer practical implications for communication skills training.
\end{abstract}

Keywords: miscarriage, early pregnancy loss, relational dialectics theory, bad news delivery 


\section{Tensions and Contradictions in Interns' Communication about Unexpected Pregnancy Loss}

Early miscarriage, defined as the loss of a pregnancy prior to 12 weeks of gestation, is a common and potentially traumatic health event. Estimates suggest that as many as 1 in 4 pregnancies ends in miscarriage (American Pregnancy Association, 2017). A miscarriage is almost always an unexpected outcome, as most women and couples anticipate a healthy pregnancy (Maker \& Ogden, 2003). The shock of a miscarriage is amplified if a woman who is asymptomatic finds out about the loss during an ultrasound at a routine prenatal appointment (Wallace, Goodman, Freedman, Dalton, \& Harris, 2010). In cases of asymptomatic pregnancy loss, physicians are faced with the complex communicative task of delivering unanticipated news while presenting the patient with a range of options for managing the miscarriage, all while the patient is in the midst of coping with this startling, and perhaps devastating, news (Olesen, Graungaard, \& Husted, 2015). Given the complicated nature of delivering the unwelcome news of an unanticipated pregnancy loss while also helping women navigate the management of a miscarriage, our goal in the current study was to investigate health care providers' communication about unexpected pregnancy loss. In the following sections, we review literature pointing to the critical importance of communication during this sort of medical encounter and outline a theoretical framework, Baxter's (2011) relational dialectics theory 2.0, for examining communication in this context.

\section{Communication and Early Pregnancy Loss}

Women are likely to feel anxious and wholly unprepared for coping with a miscarriage, particularly when they have yet to experience bodily symptoms of the loss (Maker \& Ogden, 2003; Swanson, 2000). Yet early pregnancy loss is a "preference sensitive clinical scenario," 
meaning that a patient can choose from multiple valid treatment options (Wallace et al., 2010, p. 458). Therefore, news of the loss should be communicated clearly, and a woman should be presented with the four equally viable options for managing the physical process of a miscarriage. Those options are expectant management, medical management, and two types of surgical management (Wallace et al., 2010). Expectant management consists of waiting to see whether a woman's body expels the pregnancy without medical or surgical intervention. Medical management involves the use of medication to initiate and progress the miscarriage. Finally, one surgical approach can be conducted in a physician's office and involves a local anesthesia while the other approach is performed in a hospital or surgery center and involves general anesthesia (Wallace et al., 2010). Thus, women face both digesting news of the loss and deciding how to move forward in handling the physical effects. Situations of asymptomatic pregnancy loss present a critical need for physicians to communicate the circumstances of the loss and assist women in making an informed choice (Séjourné, Callahan, \& Chabrol, 2010) while honoring the emotional state and preferences of the patient (Schreiber et al., 2016).

Although physicians in obstetrics and gynecology have argued that patients experiencing nonemergent early pregnancy loss should be able to choose from any of the four medically viable management options, research suggests that women do not always feel as though their preferences are honored by physicians and that physicians do not fully discuss the range of available options (Brann \& Bute, 2017; Olesen et al., 2015; Wallace et al., 2010). For instance, in one study, providers discussed only three of the available options and spent more time discussing surgery than other management options (Brann \& Bute, 2017). Moreover, women report feeling unsatisfied with how their health care providers talk about miscarriage (Brann, 2015; Bute, 2015), which can amplify women's distress and inhibit their coping. Women have 
described an overall sense of discontent with providers' communication (Bute, 2015) and a lack of communicative support from providers (Brann, 2015). In fact, some women have reported that providers' lack of empathy and support can intensify, rather than ameliorate, the trauma they experience (Bellhouse, Temple-Smith, Watson, \& Bilardi, 2018). Despite these findings, the existing literature tells us little about how these sorts of medical encounters unfold and the specific messages that can prompt women to leave these interactions feeling dissatisfied or further traumatized. Examining enacted communication about unexpected pregnancy loss in the clinical context could provide a better understanding of why women report feeling uninformed, frustrated, and disappointed when coping with early miscarriage and could result in practical implications for training of health care providers. In this study, we employed Baxter's (2011) updated version of relational dialects theory (which she refers to as RDT 2.0) to assess how interns (i.e., first-year residents) in obstetrics and gynecology $(\mathrm{OB} / \mathrm{GYN})$ deliver news of an unexpected early pregnancy loss and discuss options for management of the miscarriage during training simulations. In the next section, we outline the tenets of RDT 2.0 that informed our analysis.

\section{Relational Dialects Theory 2.0}

RDT 2.0 is a communication-centered framework that is an appropriate and useful theoretical lens for (a) focusing on enacted speech (Baxter, 2011) and (b) revealing "contextspecific discursive struggles" (Baxter \& Norwood, 2015, p. 280), such as those that might arise

during simulated medical encounters. Indeed, Baxter (2011) calls for increased attention to actual talk when studying interpersonal communication because a focus on enacted speech allows scholars to explore how meanings arise in the interplay of contradictory discourses (Baxter \& Norwood, 2015). A key assumption of the theory is that discursive tension is inherent to 
interpersonal communication (Baxter \& Norwood, 2015). In the case of unexpected early pregnancy loss, examination of how miscarriage is discursively constructed by interns can reveal competing and contradictory discourses that emerge during the interaction. During a traumatic loss, contradictory and competing discourses, particularly from a powerful expert like a health care provider, could influence women's comprehension of the diagnosis, their decision-making, and their coping.

Because RDT is a complex and highly detailed theory, Baxter and Norwood (2015) suggested that "it might benefit researchers to focus on a subset of its concepts and propositions in a single study" (p. 289). As such, our study of communication about unexpected pregnancy loss was guided by a specific set of RDT 2.0's theoretical assumptions, which is an approach employed in other explorations of patient-provider communication using RDT 2.0 (e.g., O'Hara, 2017; O’Hara \& Shue, 2018). First, we attend to what Baxter (2011) calls nonantagonistic struggles, which are competing discourses apparent in the utterances of one party. Because we were particularly interested in how physicians deliver and explain the diagnosis of an unexpected impending miscarriage and explicate the possible management options, we wanted to attend to the interplay of competing discourses in interns" speech to uncover the "simultaneous articulation of contradictory discourses" (O'Hara, 2017, p. 328) by a single speaker.

Baxter (2011) contends that all interpersonal talk, including communication in medical encounters is part of a larger, ongoing utterance chain. One particular link in this chain, the distal already-spoken link, suggests that broader discourses that circulate in the culture at large are necessarily invoked in our interpersonal communication (Baxter, 2011). All talk is informed by “shared cultural discourses" (Baxter \& Norwood, 2015, p. 282), or the distal already-spoken sites in the utterance chain. For example, in the case of miscarriage, distal already-spoken discourses 
that circulate in the broader culture can involve the societal silence surrounding miscarriage (Authors, in press) and culturally-specific expectations for childbearing and pronatalism, fecundity/fertility, and abortion/reproductive rights (Silverman \& Baglia, 2015). Moreover, the practice of medicine and communication in health care settings are embedded in sociocultural discourses that influence the nature of medical encounters (e.g., Lupton, 2003; Street, 2003) and include expectations about patient empowerment, preferences for action and medical procedures versus “doing nothing," and informed decision-making as an idealized outcome (Brann \& Bute, 2017). Given that communication about miscarriage inevitably summons distal already-spoken discourses, we sought to explore how these discourses are invoked by health care providers in medical encounters that involve delivering news of a miscarriage and talking through treatment options. Moreover, attention to the distal parts of the utterance chain that reflect broader societal discourses is consistent with our ongoing interest in the way speakers invoke societal expectations in interpersonal talk about miscarriage (Authors, in press).

Finally, RDT 2.0 represents a critical turn in theorizing about interpersonal communication by encouraging increased attention to discursive inequality and issues of struggle and power, allowing for deep analysis of competing discourses and discursive tensions while acknowledging that meanings of talk have an evaluative dimension (Baxter \& Norwood, 2015). More specifically, Baxter (2011) distinguishes between centripetal and centrifugal discourses that are expressed in interpersonal talk. Centripetal discourses are dominant and easily legitimated while centrifugal discourses exist in the margins and are easily delegitimatized. Power, particularly in the case of medical encounters that already involve differential power, resides in discourses and has "the discursive capacity to define reality" (Baxter, 2011, p. 124). As Lupton (2003) notes, our bodily experiences are given meaning through discursive processes. 
In the case of patient-provider interpersonal communication about unexpected pregnancy loss, we must attend to what is centered and what is pushed to the margins.

Based on tenets of RDT 2.0, we examined nonantagonistic struggles in interns' communication about early miscarriage, with special attention to discursive inequality apparent in the centripetal-centrifugal struggles at play in interns' talk. More specifically, we sought to answer the following research questions:

RQ1: What distal already-spoken discourses inform how health care providers communicate about early pregnancy loss?

RQ2: What are the competing discourses at play in health care providers' communication surrounding early pregnancy loss?

By exploring these tensions, we may be better able to understand the dissatisfaction and confusion women experience during the delivery of the bad news of a pregnancy loss and suggest ways to improve communication during these emotionally-charged encounters.

\section{Methods}

After receiving institutional review board approval, we observed 40 encounters between $\mathrm{OB} / \mathrm{GYN}$ interns and five standardized patients at a Midwestern academic medical center. We position such interactions as a useful site for attending to enacted speech about unexpected pregnancy loss because we can identify opportunities for communication skills training and intervene early in physicians' careers. Interns participate in the standardized patient encounter during an Objective Structured Clinical Examination (OSCE) at the end of their first year of residency. The residency program does not currently offer formalized training in communication about pregnancy loss, and the authors have been working with the residency director to evaluate current practices and offer suggestions for improvement. 
For the present study, both authors reviewed video recordings of 40 encounters. Of these 40 encounters, 18 were also observed live via video feed by at least one author. In addition, we observed two feedback sessions following the OSCE where interns were given feedback about their skills from an assigned practicing physician, and we took extensive fieldnotes during those sessions. A trained research assistant transcribed the patient encounters noting not only the oral communication but also nonverbal behaviors (e.g., touch, proximity, silence). The 260 singlespaced pages of typed transcripts were used for this analysis. Details of the training session and our analysis follow.

\section{Objective Structured Clinical Examination (OSCE)}

Forty OB/GYN interns (36 females, 4 males) participated in the pregnancy loss OSCE during the previous 5 years. This training technique utilizes standardized patients to improve providers' communication skills and has been shown to improve bad news delivery (Rosenbaum, Ferguson, \& Lobas, 2004). Upon approaching an examination room, interns were instructed to read a standard door note that describes a "missed AB" scenario (i.e., missed abortion or miscarriage). The standardized patient at this station is a paid, trained actor who portrays a patient who desires the pregnancy, is without her spouse, has had a previous healthy pregnancy and birth, and is unaware that she is miscarrying. She is 9 weeks pregnant, asymptomatic, and has just had a routine ultrasound and is waiting for the physician to discuss the results with her. Interns have approximately 15 minutes with the standardized patient to deliver the news of the miscarriage and explain to her the management options to help her decide on a course of treatment. At the conclusion of the OSCE, interns meet as a group with the practicing physicians who remotely observed interactions to hear feedback about their performance. Later, interns 
meet individually with a practicing physician to discuss individual comments from the physician and feedback from the standardized patient.

In a previous study where women who had experienced miscarriage observed these interactions and discussed them in a focus group setting, participants reported on the realism of the interactions and were unaware that the encounter did not involve an actual patient experiencing a miscarriage (Authors, 2018). This supports that interns engage in communication behaviors that are similar to communication patterns of providers with actual patients.

\section{Data Analysis}

Upon receiving transcripts of the patient encounters, one author read through the transcripts while listening to and watching the video files for accuracy. Then, both researchers read five transcripts to get an overall sense of what was being communicated during the interaction. After meeting to discuss emergent ideas, it was clear that inherent tensions were evident in the communication from the provider. With this idea in mind, we returned to the literature and began exploring relational dialectics theory, particularly RDT 2.0 (Baxter, 2011) and then to the data to explore the constructs conceptualized in RDT 2.0. We performed a sixstep process of thematic analysis known as contrapuntal analysis (Baxter, 2011), which allowed us to emphasize how competing discourses were evident within the patient encounter. After becoming familiar with the data set through observing encounters, reading transcripts, and discussing interactions (step 1), we began with open coding (Corbin \& Strauss, 2015) to generate initial coding categories (e.g., medicalization) (step 2). After coding an additional five transcripts, we met to discuss the independent coding. We noted several inherent tensions within the interns' utterances. Thus, we recognized the most salient construct was the interns' competing discourses at the distal already-spoken link of the chain of speech communion. We 
refined our initial coding by re-categorizing relevant tensions to note where they connected to the distal already-spoken until we reached theoretical saturation (step 3). We created a codebook from this process, which we refined during our weekly discussions about the data and its fit to the theoretical framework. To assist with recognizing the tensions, we looked for discursive markers described by Baxter (2011): negating (rejection of a competing discourse with words such as "no," "never," and "not"), countering (replacement of an expected discourse with words such as "just," "but," and "however"), and entertaining (recognition of multiple discourses with words such as "may," "could," and "likely"). We used the refined codebook to code the remaining 30 transcripts to assess if the themes were replicated throughout the data set (step 4). Once completed, we met to discuss our findings and to finalize and conceptually define the observed discourses (step 5). Confident in our findings, each researcher located exemplars from the data and added them to the codebook as representative of the evident themes (step 6).

\section{Results}

Our analysis revealed two primary distal already-spoken discourses invoked by interns: discourses of medicalization and discourses of rationality and informed consent. Within each of these primary themes are supporting subthemes that elucidate the communicative tensions inherent in these simulated medical encounters.

\section{The Discursive Medicalization of Miscarriage}

Although women have found ways to circumvent the patriarchal, hegemonic, politicized medical industry by providing care for themselves and other women, it is clear that women's health issues, particularly those related to sexual health and reproduction, are often the domain of the medical establishment. Pregnancy, childbirth, menstruation, and menopause have been socially constructed as conditions that are medicalized, meaning that they are often defined as 
medical in origin and, therefore, require medical solutions (Lorber \& Moore, 2002; Lupton, 2003). As such, miscarriage is socially constructed as falling under the purview of health care professionals, requiring diagnosis, management, and treatment (Bellhouse et al., 2018); and physicians' communication about pregnancy loss unavoidably invokes expectations that the loss is medical in nature and, therefore, medical in solution. In addition to discursively constructing miscarriage as a medical condition, interns also communicated confusing information about the diagnosis as both normal and abnormal, (un)certainty about the cause of the miscarriage, unequal treatment of management options, and contradictory medical terminology.

A natural and common abnormality. In delivering the diagnosis, interns simultaneously described miscarriage both as a common and natural incident and also as an abnormal, pathological occurrence, suggesting that while pregnancy loss is a clinical event that requires medical attention, it is also a somewhat ordinary event. As Bellhouse and colleagues (2018) noted, framing miscarriage as a normal and routine pregnancy complication is evidence of the medicalization of pregnancy loss. Discursive attempts to normalize miscarriage largely focused on framing pregnancy loss as something many pregnant women experience, even though the topic is rarely discussed. For example, one intern stated,

Miscarriages are very common this early in pregnancy, okay? They happen in up to $20 \%$ of pregnancies and the problem is, it's something that women don't really talk about so you're not alone in this situation, okay? It's just something that people usually just keep to themselves and that makes it difficult.

Another intern, who did not discuss specific statistics of commonality, still reaffirmed the routine nature of miscarriages by saying, "Miscarriages just happen sometimes. They're actually more common than people think because people don't, you know, go around telling other people that 
they've miscarried." Interns typically used the term "common" and sometimes attempted to quantify that commonality by stating " $20 \%$ " or "about 1 in 5 " pregnant women have a miscarriage.

While trying to normalize early pregnancy loss by emphasizing how common it is, interns also, perhaps unwittingly, normalized the societal silence that surrounds miscarriage (also see Authors, in press) by frequently mentioning that although miscarriage occurs regularly, most people don't talk about it. Thus, they reinforced the distal already-spoken discourses that mark miscarriage as a taboo topic.

Interns also framed pregnancy loss as a natural bodily process while concurrently describing the cause of the miscarriage, and sometimes the miscarriage itself, as an abnormality. As Foster (2005) noted, the term "miscarriage" is inextricably linked to the suggestion of an aberration or an irregularity because it implies a woman mis-carried her unborn child. When interns used the term "miscarriage" in delivering the news, they immediately implied abnormality. However, interns described miscarriage as a "normal" and "natural" process, as "a common and natural thing that happens." At the same time, interns also made it abundantly clear that the pregnancy and/or the baby were not normal. They used phrases such as, “there wasn't something right with the baby," "the baby's genetics could've caused it to not be normal and sometimes that can't grow normally," "it's just not a normal pregnancy," and, "when something like this happens, the pregnancy wasn't normal to begin with." Thus, women are told that what they are facing is natural and common but not normal. As one intern put it, "You know it's usually just, um, the body’s sort of natural way of, you know, removing an abnormally developing pregnancy." The normalizing of an "abnormal" event highlights one of many nonantagonistic struggles in interns' communication with patients. 
(Un)certain causes. Although pregnancy loss is framed as a pregnancy complication that requires medical solution, interns in our study typically struggled to pinpoint a precise cause for the miscarriage. They explained, "we don't know why this happens," "it's difficult to tell exactly what was the issue," "we don't have a reason for it," and "we may never know why this happened.” One intern said, "Do you have any questions, anything that I...I probably won't be able to answer questions as far as why this happened."

At the same time that they assured patients that the cause of the miscarriage would remain undetermined, interns expressed certainty the patient was not to blame with most of them telling the patient, "It's nothing you did." Interns used negating language that acknowledges then rejects competing discourses (Baxter, 2011). Although interns were unsure about what precipitated the miscarriage, they expressed certainty that the patient was not at fault. In doing so, they alluded to distal already-spoken discourses that blame women for miscarriage. Research by Bardos, Hercz, Friedenthal, Missmer, and Williams (2015) revealed widespread misunderstanding about causes of miscarriage, with survey respondents incorrectly indicating that stress, lifting heavy objects, and other lifestyle choices were primary causes of pregnancy loss. Our analysis suggested that almost all interns sought to reject such discourses by emphasizing that the patient did nothing to cause the loss. Although such expressions are likely meant to reassure the patient, they also suggest that the patient has no control to prevent a future loss. Interns said, “there's nothing you could have done differently," "there's nothing you could've done," and "there's nothing that you could've changed to prevent it from happening; it's just something that happens." The competing talk about uncertainty intermingled with certainty could be particularly confusing when reassurances are tentative in nature, like this one: 
“It's usually nothing you've done. It's probably something that couldn't have been prevented. And I think you were probably doing a great job and I don't think it's anything that you did."

Expectant management as marginalized. Another clear example of the medicalization of pregnancy loss is apparent in the way interns described the options for managing a miscarriage. Expectant management was repeatedly and consistently marginalized as the least preferred option, while surgery under general anesthesia was presented as the fastest and most definitive option. The language that interns used to discuss the management options highlights a centripetal-centrifugal struggle in which the least invasive and least medicalized option is discursively marginalized while the most invasive option, surgery under general anesthesia, is centered.

Although expectant management was almost always mentioned first in describing the options for managing the impending miscarriage, it was also described as a passive process. Interns in every encounter trivialized the physical experience of expectant management by referring to it as "doing nothing," "you just wait," "just go home, wait, and do nothing," "not doing anything," or "just waiting." By describing expectant management as "doing nothing," interns neglected the active embodied process a woman's body endures during a miscarriage (see Bute \& Brann, 2015) while simultaneously implying that choosing to forgo medical intervention is the equivalent of choosing to do nothing at all.

In contrast, surgical management, specifically the surgical option that requires general anesthesia and a procedure in a hospital or surgery center (i.e., a dilation and curettage or D\&C), was presented as an active alternative that resolves the medical issue quickly. Notice, for instance, the contrast in how the intern describes expectant and surgical management in the following excerpt: 
The first option is not doing anything. It's usually not something we recommend. I always like to warn people that it could cause pretty heavy vaginal bleeding, pretty heavy cramping so it can be pretty uncomfortable... With D\&C, this is one of the accurate ways of removing all the products without having to do any further intervention.

Similarly, another intern said, "Most women tend to choose either the medicine or the surgery just so that it could be done with and kind of off their mind instead of waiting around." Yet another intern informed the patient that a D\&C is a "quick procedure" and that "If you were to pass (the pregnancy) on your own then you would potentially have more bleeding than you would from the surgery."

In addition to discursive moves that framed expectant management as a passive process, it was also framed as a risky choice and a lengthy process that would likely necessitate eventual medical intervention. One intern explained, “And then if we wait, there's risk of infection and we could inevitably end up having to bring you back in because if over the next -- it can take up to a month in some people." Similarly, another intern said, "Sometimes that doesn't work, and you would need further medicine or also go get a procedure as well. There's still a possibility that you could have to have future procedures."

Although the lingering possibility of future medical interventions was emphasized most frequently in discussions of expectant management, some interns suggested that all options (expectant, medical, and surgical), carry the risk of additional intervention down the road:

With expectant management with letting you go home, um, and to see if you are to miscarry on your own, the risk is that it wouldn't, that you would need a procedure to eventually take care of it. There's a risk, um, of infection, um, if it were to stay for too long, okay? With the medicine, there's also a risk that we give you the medicine, you 
have the cramping and bleeding, pass some of the pregnancy but not all of it so then you would maybe need another procedure.

Medical intervention was inarguably emphasized by interns as the favored resolution for managing an impending miscarriage and as a possible outcome regardless of which alternative a patient might choose. Discursive inequality constructed surgery as the centripetal and preferred option. Other options (e.g., expectant management) were not only marginalized but also presented with inaccurate information, which likely influenced decision-making.

Contradictions in terminology. During the course of the encounter with the standardized patient, interns changed the terminology they used to reflect an increasing emphasis on miscarriage as a medical problem requiring medical intervention. During the delivery of the unexpected news near the beginning of the interaction, interns used terms like "baby" or "pregnancy" to explain the situation at hand. For example, one intern said, "So, basically what we saw on the ultrasound was that your baby no longer has a heartbeat. Um, and so what that means is that the pregnancy probably is not going to progress any further." Another intern simply said, "Your baby has died." During the course of the interaction, most interns changed their language as they described the options for expectant, medical, or surgical management. Instead of referring to a baby, interns shifted their terminology to refer to "contents," "products," "parts," “tissue," or even "it." In one example, an intern explained expectant management: "The first option is expectant management, or just waiting. In many cases if you wait a few weeks, your body will naturally dilate and expel what's inside. That would be natural passing of tissue." In detailing the process of surgical management, one intern said, "which is a little bit more aggressive where, you know, we would go in and then take out the contents" while another explained, "We would, um, pull out or scrape out everything that's in there - the, the tissue." 
Interns predictably changed their language over the course of the interaction to depersonalize the process and underscore the miscarriage as a medical concern.

\section{The Discursive Construction of Rationality}

All medical encounters are embedded in a larger political/legal context in which physicians are expected and obligated to disclose the full range of treatment or management alternatives, as well as the risks and benefits of each option so that patients can make informed decisions (Street, 2003). Moreover, physicians are required to obtain written consent from patients before performing medical procedures (Donovan et al., 2014). Implicit within such legal obligations is a discourse of rationality (Baxter, 2011) suggesting that when patients are fully informed of the range of options available to them, they will reason through these options and make a rational choice about the best course of action. In the U.S., "the discourse of rationality is taken for granted as the natural way to understand human action" (Baxter, 2011, p. 58).

However, patients' interpretations of risks and informed consent conversations are often marked by uncertainty (Donovan et al., 2014; Donovan-Kicken et al., 2012). Based on our analysis of OSCE interactions in this study, we contend that competing discourses about the likelihood of risk and the possibility of future pregnancies could be confusing for patients. When faced with a potentially devastating loss, expecting patients to make a rational decision void of emotion is an unlikely task.

Inconsistent framing of risk. The instructions for the OSCE examined in this study guided interns to tell the patient she was experiencing a miscarriage and then to counsel the patient on her options. All interns took these instructions to mean that they should explain the risks and benefits of the available management options. Within these explanations are contradictory messages about the nature and likelihood of risk. 
Discussions of risk were most extensive and incongruous when interns presented surgical alternatives for managing the miscarriage. Risk was simultaneously framed as "low, but something that could happen," and as "big," yet "small:"

$\mathrm{Um}$, the big risk is putting instruments at the top of the uterus is something called perforation, which is making a hole in the uterus. The risk of that is quite small. Um, however if that did happen, you may or may not have to stay overnight for us to observe you and make sure everything's okay. Like I said, the risk of that is small.

In some cases, interns minimized the likelihood of a particular risk by communicatively framing risks as events that are unlikely to occur but that they "have to bring up:"

Okay, and the biggest risk is that you would have bleeding that we couldn't control and we would have to take your uterus. I have never seen this happen in person, but it is a risk that we have to tell you about, okay?

Contradictions were often communicated as the encounter unfolded. For example, one intern discussed surgical management as the most effective option by explaining that, "there are some risks to it, but the benefits are one that you wouldn't be passing it at home, wouldn't be having all the cramping and the bleeding and sitting on the toilet or anything like that." Then later in the encounter, the intern elaborated on the possible risk of surgery:

And any time that we do a procedure that involves the cervix or the uterus itself, there's the possibility of needing to do a hysterectomy if the bleeding is too much and we can't control it. Um, this is not something that we anticipate to happen with this procedure, but I always have to bring it up because it is a possibility.

Thus, surgery is framed as a safer alternative to bleeding at home yet at the same time includes a risk of so much bleeding that a hysterectomy could be necessary. Thus, the contradictory 
information about risk for the options presented likely left patients confused and uninformed for making a decision.

Future (im)possibilities. Many interns tempered the bad news of an imminent miscarriage by assuring the patient that she could get pregnant again the future. In fact, the possibility of a future successful pregnancy was mentioned frequently even before the standardized patient inquired about the likelihood of another pregnancy. In other words, interns used countering language (Baxter, 2011) to supplant the expectation that future pregnancies would be problematic with assurances that the patient could have a successful pregnancy in the future. Interns often mentioned a future pregnancy right after the delivery of the bad news by stating things such as "Well the good news is that you can try again, okay?" "Some women early after miscarriage can get pregnant again and have a normal baby after pregnancy," or “Oftentimes this happens and we don't know why. However, on the other side of things, most women after having a miscarriage will go on to having a successful delivery of a baby." As such, the interns began addressing the future even before they had fully addressed the current situation.

Some interns then went on to explain that the current miscarriage may or may not mean that the patient faced an increased risk of a subsequent miscarriage. "In a future pregnancy, it would be the same amount of risk - slightly higher, but not in a significant way," explained one intern. Another said, "There is a slight increased risk of miscarriage on subsequent pregnancies but it's minimal." And finally:

You are maybe at a slightly higher risk at having a miscarriage compared to the average woman who has never had one, um, but you should still have an equally good chance and be able to get pregnant and have a baby, have a normal, healthy baby again, okay? 
Within the same sentence, this intern tells the patient that she has a slightly higher risk of future miscarriages than other women but also an equal chance of having a "normal" baby.

After assuring the patient that she can still get pregnant in the future early in the interaction, interns then went on to explain that complications or risks of surgery, the discursively preferred alternative, could impede the ability to get pregnant in the future. As one intern said,

One thing I didn't mention about the procedure that I'll talk about now is that while we're in there, kind of scraping around on the uterus, there's a possibility that some scar tissue could form and that could possibly lead to the prevention of a future pregnancy.

And another explained, "There's a risk that the scar could make it difficult to get pregnant again in the future." So not only is risk discussed inconsistently, it is also constructed as a looming threat to future desires.

\section{Discussion}

As Street (2003) noted, a medical encounter is fundamentally an interpersonal conversation. Although Baxter (2011) largely frames RDT 2.0 as a framework for examining relational talk in marriages, friendships, and family relationships, our work contributes to a growing body of research that draws on dialectical sensibilities to explore patient-provider communication (O’Hara 2017; O’Hara \& Shue, 2018; Olufowote, 2011). RDT 2.0 is especially appropriate for uncovering overt and covert meanings in these value-laden interactions, as “experiences are given meaning through communication” (Baxter \& Norwood, 2015, p. 282). Women who are in the midst of a miscarriage are forced to make sense of what is occurring with their bodies, to decide the best course of treatment, and to assign meaning to the entire experience, which is done, in part, through communication with their health care providers. As 
Baxter and Norwood (2015) highlight, RDT 2.0 is not aimed at predicting generalizable findings but is instead intended to assess the "intelligibility of situational communication" (p. 280). The meaning-making process of this situation, communication of an impending miscarriage, is heavily influenced by the discourse of the provider, and the nonantagonistic struggles therein, when relaying the bad news and explaining the various management options. This can be especially problematic in this context because complications for surgical interventions (e.g., scar tissue), in rare cases, can contribute to greater risk of future miscarriages or pregnancy complications (Hooker et al., 2014), which could then lead to more medical interventions in the future. Physicians are often trained from a biomedical perspective to "fix" the physical problem without taking into consideration how their "solution" might affect other areas of a patient's health and life (Longino, 1997). This linear approach to thinking about, and practicing, medicine does not account for systematic effects, which likely leads to several of the contradictions noted in the interns' communication.

Given that nonemergent early miscarriage, like the scenario used in the OSCE, is a scenario in which the patient's preferred management option should prevail (Wallace et al., 2010), it is especially problematic that competing discourses could affect the sensemaking and decision-making processes for women. As Baxter (2011) noted, these discourses are oftentimes not given equal value. The marginalization of expectant management, in particular, was evident in the interns' communication reinforcing how biomedical approaches were privileged (Lupton, 2003). Moreover, because discourses are fluid (Baxter, 2011), the tension inherent in the interns' speech became evident as they fluctuated between competing discourses. For example, the interns clearly illustrated such fluctuations when discussing risk as both "big" and "small" or referring to both "the baby" and the "contents," as evidenced in our results. 
Interns in our study clearly privileged the Voice of Medicine over the Voice of the Lifeworld (Mishler, 1984) by favoring discourses of the medicalization of miscarriage and rationality. The distal already-spoken utterances reflected their medical training, which focuses on a biomedical approach to health, as well as broader societal views, which privilege silence around reproductive health, particularly about miscarriage. As we noted in an earlier study (Authors, in press), individuals invoke societal expectations about interpersonal talk, or lack thereof, surrounding miscarriage. This study reveals that these broader "shared cultural discourses" (Baxter \& Norwood, 2015, p. 282) that circulate within a culture are also occurring in exam rooms by health care providers even when delivering diagnosis and treatment options to a woman in the midst of her loss. Our analysis is consistent with previous work suggesting that women do not always feel as though their preferences for managing a miscarriage are honored or understood by physicians (Olesen et al., 2015; Rowe, 2014; Wallace et. al, 2010). Perhaps the contradictory language invoked by providers contributes to women's feelings of neglect. If an asymptomatic patient facing an impending miscarriage is told that her body is experiencing something natural that is also pathological, then it stands to reason that she might feel conflicted about which alternative would be best for her.

At the same time, tensions and contradictions are not always inherently problematic. For instance, Authors (2018) reported that women preferred for interns to use medical language (e.g., tissue, contents) to describe the details involved in surgical management of miscarriage. They do not want to hear that a "baby" would be removed from the uterus; they prefer more medicalized terminology for that portion of the discussion. However, when receiving the initial bad news, women prefer for the physician to recognize the gravity of their loss by noting that they had lost a baby, not simply "tissue" or "contents." Although our findings suggest that interns framed 
expectant management as the least preferred option, we want to be clear that we recognize expectant management is not the preferred option for every woman and that many women might find medicalized treatments, including surgery, to be the best option for them.

\section{Practical Implications}

Our results offer practical implications for training $\mathrm{OB} / \mathrm{GYN}$ interns by drawing attention to the ways that contradictory presentations of treatment options and the risks therein could potentially confuse patients and compound their distress (Bellhouse et al., 2018). In addition, training programs should emphasize that patient preferences should take precedence in cases of nonemergent, uncomplicated pregnancy loss (Wallace et al., 2010). As such, providers should be trained to evaluate their own communication to recognize both subtle and explicit language that could lead women to assume that some management options are "better" than others, which could help ensure that women's own preferences are not ignored or denied (Olesen et al., 2015).

One thing that may be particularly useful for health care providers is simply the recognition of distal already-spoken discourses of medicalization and rational decision-making and how these broader discourses influence, and contradict, the information they provide to their patients. Sensitivity training could increase awareness among health care providers, and communication skills training could educate providers on effective ways to provide noncontradictory, accurate information to women who are experiencing a miscarriage and must make a decision for managing that experience. Identifying how these communicative patterns begin in residency and prompting providers to reflect on their language during OSCE encounters could give them an opportunity to change the way they talk about pregnancy loss as they move toward becoming independent practicing physicians. 


\section{Limitations and Directions for Future Research}

Our study focused on only one aspect of the utterance chain, as suggested by Baxter and Norwood (2015), but it may be beneficial to explore other constructs of RDT 2.0 to expand our knowledge about competing discourses health care providers use when communicating diagnoses and treatment options. This could be particularly fruitful when observing interactions between providers and patients with whom they have developed a clinical relationship, such as research by O'Hara (2017) in the diabetes context. For example, exploring proximal alreadyspokens could lead to greater understanding of how potentially competing interpersonal discourses also influence understanding and sensemaking.

Not only would focusing on existing relationships be beneficial to explore competing discourses, but it would also provide insight into communicative behaviors enacted in real-time and real spaces. Although the standardized patient scenario presented in this study was shown to be realistic (Authors, 2018), interns know that they are being observed and will be given feedback, which may alter how they perform in a patient encounter. For example, interns may have been nervous and therefore, communicated in contradictory ways. Still, we feel confident that the competing discourses evidenced in this study are representative of the communicative practices by other health care providers in similar situations. Moreover, studying simulated encounters allowed a close analysis of enacted speech without posing the ethical dilemmas involved in recording or observing naturally occurring conversations in which a health care provider delivers news of an imminent miscarriage.

It could be useful to assess the similarities and differences among health care providers to determine other influences affecting the communicative tensions evident in the diagnostic speech. This study was limited, for example, by the number of male interns in the sample. With 
only four male participants, it was not possible to assess any gendered differences that may be inherent in the speech acts surrounding an experience that is embodied by women (Bute \& Brann, 2015).

Another insightful approach would be to explore the narratives of women in similar patient encounters to assess if, and how, they reframe the discursive inequality inherent in the discourse. It might prove insightful to assess the transformative struggle and their methods for reframing competing discourses so they are no longer competing (Baxter, 2011). By doing so, new insight can be gleaned as to how that new perspective allows women to make sense of the information to cope with crises and "turn the calamities of fate into the gifts of humanity" (Bochner, Ellis, \& Tillmann-Healy, 1998, p. 53).

Finally, it may be useful to apply RDT 2.0 to other "preference sensitive clinical scenarios" (Wallace et al., 2010, p. 458) that require decision-making, such as infertility treatment options. Similar to the miscarriage context, individuals struggling with infertility issues may experience comparable discursive tensions given the medicalization of infertility treatment options and societal silence of infertility embedded within broader cultural discourses (Jensen, 2016). Examining other contexts can provide insight to the inherent practices of health care providers when delivering uncomfortable news about culturally taboo topics. 


\section{References}

American Pregnancy Association. (2017). Miscarriage. Retrieved from http://www.americanpregnancy.org/pregnancy-complications/miscarriage.html

Bardos, J., Hercz, D., Friedenthal, J., Missmer, S. A., \& Williams, Z. (2015). A national survey on public perceptions of miscarriage. Obstetrics \& Gynecology, 125, 1313-1320.

Baxter, L. A. (2011). Voicing relationships. A dialogic perspective. Thousand Oaks, CA: Sage.

Baxter, L. A., \& Norwood, K. (2015). Relational dialectics theory. In D. O. Braithwaite \& P. Schrodt (Eds.), Engaging theories in interpersonal communication ( $2^{\text {nd }}$ ed.). Thousand Oaks, CA: Sage.

Bellhouse, C., Temple-Smith, M., Watson, S., \& Bilardi, J. (2018). “The loss was traumatic... some healthcare providers added to that": Women's experiences of miscarriage. Women and Birth. https://doi.org/10.1016/j.wombi.2018.06.006

Brann, M. (2015). Nine years later and still waiting: When health care providers' social support never arrives. In R. Silverman \& J. Baglia (Eds.), Pregnancy loss: A narrative collection (pp. 19-31). New York, NY: Peter Lang Publishing.

Brann, M., \& Bute, J. J. (2017). Communicating to promote informed decisions in the context of early pregnancy loss. Patient Education and Counseling, 100, 2269-2274.

Bochner, A.P., Ellis, C., \& Tillmann-Healy, L. (1998). Mucking around looking for the truth. In B. M. Montgomery \& L. A. Baxter (Eds.). Dialectical approaches to studying personal relationships (pp. 41-62). Mahwah, NJ: Lawrence Erlbaum.

Bute, J. J. (2015). Honoring stories of miscarriage: A plea to health care providers. In J. Baglia and R. Silverman (Eds.). Pregnancy loss: A narrative collection. (pp. 33-44). Peter Lang Publishing. 
Bute, J. J., \& Brann, M. (2015). Co-ownership of private information in the miscarriage context. Journal of Applied Communication Research, 43, 23-43.

Corbin, J., \& Strauss, A. (2015). Basics of qualitative research $\left(4^{\text {th }}\right.$ ed.). Thousand Oaks, CA: Sage.

Donovan, E. E., Crook, B., Brown, L. E., Pastorek, A. E., Hall, C. A., Mackert, M. S., \& Stephens, K. K. (2014). An experimental test of medical disclosure and consent documentation: assessing patient comprehension, self-efficacy, and uncertainty. Communication Monographs, 81, 239-260.

Donovan-Kicken, E., Mackert, M., Guinn, T. D., Tollison, A. C., Breckinridge, B., \& Pont, S. J. (2012). Health literacy, self-efficacy, and patients' assessment of medical disclosure and consent documentation. Health Communication, 27, 581-590.

Foster, E. (2005). Desiring dialectical discourse: A feminist ponders the transition to motherhood. Women's Studies in Communication, 28, 57-83.

Hooker, A. B., Lemmers, M., Thurkow, A. L., Heymans, M. W., Opmeer, B. C., Brölmann, H. A. M., Mol, B. W., \& Huirne, J. A. F. (2014). Systematic review and meta-analysis of intrauterine adhesions after miscarriage: prevalence, risk factors, and long-term reproductive outcome. Human Reproduction Update, 20, 262-278.

Jensen, R. E. (2016). Infertility: Tracing the history of a transformative term. University Park, PA: Penn State Press

Longino, C. F. (1997). Beyond the body: an emerging medical paradigm. American demographics, 19, 14-19.

Lorber, J., \& Moore, L. J. (2002). Gender and the social construction of illness. Lanham, MD: Rowman \& Littlefield. 
Lupton, D. (2003). Medicine as culture: Illness, disease and the body in Western societies. London: Sage Publications.

Maker, C., \& Ogden, J. (2003). The miscarriage experience: More than just a trigger to psychological morbidity? Psychology and Health, 18, 403-415.

Mishler, E. G. (1984). The discourse of medicine: Dialectics of medical interviews (Vol. 3). Norwood, N.J.: Ablex Publishing Corporation.

O’Hara, L. L. S. (2017). Discursive struggles in “diabetes management”: A case study using Baxter's Relational Dialectics 2.0. Western Journal of Communication, 81, 320-340.

O’Hara, L. L. S., \& Shue, C. K. (2018). Discursive struggles in 'diabetes management': Examining the proximal not-yet-spoken link. The Qualitative Report, 23, 1282-1300.

Olesen, M. L., Graungaard, A. H., \& Husted, G. R. (2015). Deciding treatment for miscarriageExperiences of women and health care professionals. Scandinavian Journal of Caring Sciences, 29, 386-394.

Olufowote, J. O. (2011). A dialectical perspective on informed consent to treatment: An examination of radiologists' dilemmas and negotiations. Qualitative Health Research, 216, 839-852.

Rosenbaum, M. E., Ferguson, K. J., \& Lobas, J. G. (2004). Teaching medical students and residents skills for delivering bad news: a review of strategies. Academic Medicine, 79, 107-117.

Rowe, D. (2015). Cruel optimism and the problem with positivity: Miscarriage as a model for living. In R. Silverman \& J. Baglia (Eds.), Pregnancy loss: A narrative collection (pp. 259-265). New York, NY: Peter Lang Publishing. 
Schreiber, C. A., Chavez, V., Whittaker, P. G., Ratcliffe, S. J., Easley, E., \& Barg, F. K. (2016). Treatment decisions at the time of miscarriage diagnosis. Obstetrics and Gynecology, $128,1347-1356$.

Séjourné, N., Callahan, S., \& Chabrol, H. (2010). Support following miscarriage: What women want. Journal of Reproductive and Infant Psychology, 28, 403-411.

Silverman, R. E., \& Baglia, J. (2015). Introduction: The politics of pregnancy loss. In R. E. Silverman \& J. Baglia (Eds.) Communicating pregnancy loss: Narrative as a method for change (pp. 1-16). New York, NY: Peter Lang Publishing, Inc.

Street, R. L. (2003). Communication in medical encounters: An ecological perspective. In T. L. Thompson, A. M. Dorsey, K. I. Miller, \& R. Parrot (Eds.), Handbook of Health Communication (pp. 63-89). Malwah, NJ: Lawrence Erlbaum.

Swanson, K. M. (2000). Predicting depressive symptoms after miscarriage: A path analysis based on the Lazarus paradigm. Journal of Women's Health \& Gender-Based Medicine, 9, 191-206.

Wallace, R. R., Goodman, S., Freedman, L. R., Dalton, V. K., \& Harris, L. H. (2010). Counseling women with early pregnancy failure: utilizing evidence, preserving preference. Patient Education and Counseling, 81, 454-461. 